\title{
Fusarium ershadii sp. nov., a Pathogen on Asparagus officinalis and Musa acuminata
}

\author{
Moslem Papizadeh • Anne D. van Diepeningen • \\ Hamid Reza Zamanizadeh • Farkhondeh Saba • \\ Hossein Ramezani
}

Accepted: 14 December 2017 / Published online: 15 January 2018

(C) The Author(s) 2018. This article is an open access publication weeks in Asparagus officinalis seedlings. In comparison mild disease symptoms were observed by the same strains on Musa acuminata seedlings.

Keywords Fusarium solani species complex. Asparagus officinalis pathogen $\cdot$ Musa acuminata pathogen

\section{Introduction}

Fusiform or banana-shaped multicelled conidia are probably the best known characteristic of the large Ascomycete genus Fusarium. Within the genus we find many plant pathogens, saprobes, mycotoxin producers, and an increasing number of human pathogens (e.g. Salah et al. 2015; van Diepeningen et al. 2014). For a long time, sections were recognized within the genus based on morphological characters. Nowadays, subdivisions within $F u$ sarium are made in species complexes consisting of sibling species with limited to no morphological variation, which can be best discriminated based on sequence data. A plea has been made to keep most of the Fusarium species complexes of these agriculturally and for human health important species under the well-known genus denominator Fusarium rather than splitting the genus in nine or more different genera (Geiser et al. 2013).

One of the more basal clades within the genus $\mathrm{Fu}$ sarium according to Geiser et al. (2013) is the Fusarium solani species complex (FSSC), centered on recently epitypified Fusarium solani (Schroers et al. 2016). However, Lombard et al. (2015) revisited various

Plants Bank, Iranian Biological Resource Center (IBRC), Academic Center for Education, Culture and Research (ACECR), Tehran, Iran 
genera of Nectriaceae and suggested renaming the Fusarium solani species complex as Neocosmospora (Lombard et al. 2015), but we prefer to use Geiser's proposal for a large Fusarium genus including virtually all Fusarium species of importance in plant pathology, medical mycology, mycotoxicology and basic research, and thus better recognized (Geiser et al. 2013). Members of FSSC are capable of causing disease on many agricultural important crops - often foot and root rots - and are the most commonly observed etiological agents of human fusarioses (Coleman 2015; O'Donnell et al. 2008).

Within the plant pathogenic fusaria it is common to talk about formae speciales describing the host plant species of an isolate. Host-specific virulence factors that determine the host or host range are usually located on dispensable supernumerary chromosomes. Within the Fusarium oxysporum species complex, host specificity and these supernumerary chromosomes were found to have been horizontally exchanged between different lineages and species (Baayen et al. 2000; Ma et al. 2013). However, formae speciales in FSSC seem to correspond to biologically and phylogenetically distinct species (Coleman 2015).

Based on multi-locus sequence analyses of core genome genes and regions, dozens of different phylogenetic lineages within the FSSC can be recognized (O'Donnell et al. 2008; Short et al. 2013; Zhang et al. 2006). Slowly, more of these lineages are described with Latin binomials and/or with more data regarding their ecological niches are published. Examples are the recent description of Fusarium petroliphilum (FSSC clade-1) and F. keratoplasticum (FSSC clade -2) (Short et al. 2013) and the epitypification of the potato dry rot pathogen $F$. solani sensu stricto (FSSC-clade-5) (Schroers et al. 2016). Fusarium keratoplasticum, a common inhabitant of soil, drainage systems and other antropogenic substrates, was recently suggested to be recombining, potentially even via heterothallic sex (Short et al. 2013; Short and O'Donnell 2014). A remarkable finding as ' $F u$ sarium solani' is generally considered homothallic or asexual (O'Donnell et al. 2008).

In this paper we describe a new species within FSSC that also forms a monophyletic clade based on multiple loci. The strains were isolated from the monocots asparagus and banana and proved especially pathogenic on the first host. The new species was characterized morphologically and phylogenetically.

\section{Materials and methods}

Macroscopic and microscopic morphology

Morphological characteristics and growth rates were studied on potato dextrose agar (PDA), synthetic nutrient agar (SNA), and carnation leaf agar (CLA). Digital images of the colonies were documented and studied after 7 days of incubation at $25^{\circ} \mathrm{C}$, with and without UV (longer incubation up to a month was considered if needed). Inoculations were performed using a dense inoculum stock which was prepared from a 10-day-old colony on CLA medium. Macroscopic properties were studied on 10-day-old colonies. Slide cultures were mounted in a droplet of lactic acid or water to be studied with an Olympus BX51 microscope equipped with a DP25 digital imaging camera. Size of various structures was determined by averaging the measurements of 2530 samples of each structure (Short et al. 2013).

\section{Growth rates}

Single conidia of isolates, grown on Carnation Leaf Agar (CLA) (Fisher et al. 1982), were transferred to the center of $8.5 \mathrm{~cm}$ Potato Dextrose Agar (PDA) and Oatmeal agar (OA) plates and incubated in growth chambers at $25^{\circ} \mathrm{C}$ and $30^{\circ} \mathrm{C}$. After $72 \mathrm{~h}$, colony diameters were measured using a ruler and the average growth rate per isolates was calculated and expressed as colony growth rate per $24 \mathrm{~h}$. Additionally, cardinal growth temperatures were determined on PDA plates that were mid-point inoculated and incubated at $5,10,15,20,25,28,30,34,37$ and $40^{\circ} \mathrm{C}$ for 7 days and any hyphal growth was studied under the light microscope (objective lenses $4 \mathrm{X}$ and 10X). The colony diameter was measured after 7 days (Hujslová et al. 2013; Selbmann et al. 2008).

Also, PDA and MEA media of different acidity ( $\mathrm{pH} 3-8.5$ ) were prepared in duplicate using a $2 \mathrm{M}$ stock solution of $\mathrm{HCl}$ or $\mathrm{NaOH}$ (Hujslová et al. 2013; Selbmann et al. 2008). Plates were inoculated (singlepoint) and incubated at $28{ }^{\circ} \mathrm{C}$ for 10 days and diameter of the colonies was measured (Hujslová et al. 2013; Selbmann et al. 2008).

Primers for molecular identification and phylogenetic analysis

Primers ITS1 (TCCGTAGGTGAACCTGCGG) and ITS4 (TCCTCCGCTTATTGATATGC) were used to 
amplify the ITS fragment (approximately $600 \mathrm{bp}$ ), primers EF1 (ATGGGTAAGGARGACAAGAC) and EF2 (GGARGTACCAGTSATCATGTT) were used to amplify a nearly $720 \mathrm{bp}$ fragment of the coding gene for $E F-1 \alpha$, primers RPB2-5F (GAYGAYMGWGATCAYTTYGG) and RPB2-7R (CCCATWGCYTGCTTMCCCAT) were used to amplify a nearly $1200 \mathrm{bp}$ fragment of the coding gene for RPB2 (Geiser et al. 2013,; O'Donnell et al. 2007, O'Donnell et al. 2008.; Short et al. 2011; Zhang et al. 2006).

DNA extraction and polymerase chain reaction

DNA was extracted using a manual purification procedure as described (Papizadeh et al. 2017a,; Saba et al. 2016). All the PCR amplifications were performed in a MyCycler ${ }^{\mathrm{TM}}$ thermal cycler system (BIORAD, USA). The $50 \mu \mathrm{l}$ PCR mixtures were prepared with $1 \mu \mathrm{LNA}$ suspension, $5 \mu$ l of PCR buffer (Fermentas), $10 \mathrm{mmol}$ of dNTPs, $2.5 \mathrm{mMMgSO} 4$, and 10 pmole of each of the primers, $5 \mathrm{U}$ of $P F U$ DNA polymerase, $0.5 \mu \mathrm{l}$ of absolute DMSO, and appropriate volume of DDW. A hotstart procedure $\left(3 \mathrm{~min}, 94^{\circ} \mathrm{C}\right)$ was used before the enzyme addition to prevent nonspecific annealing of the primers. All the PCR reactions for amplification of the ITS, and EF- $1 \alpha$ fragments entailed 35 cycles $\left(94^{\circ} \mathrm{C}\right.$ for $45 \mathrm{~s}, 56^{\circ} \mathrm{C}$ for ITS $\left[50^{\circ} \mathrm{C}\right.$ for EF- $1 \alpha$ ] for $50 \mathrm{~s}, 72^{\circ} \mathrm{C}$ for $95 \mathrm{~s}$, plus one additional cycle with a final $7 \mathrm{~min}$ chain elongation). For amplification of the selected fragment of the RPB2 gene the PCR conditions included: (1) hot start with $95^{\circ} \mathrm{C}$ for $5 \mathrm{~min}$; (2) 30 cycles of $1 \mathrm{~min}$ at $95^{\circ} \mathrm{C}, 2 \mathrm{~min}$ at $55^{\circ} \mathrm{C}$ ( or $50^{\circ} \mathrm{C}$ ), an increase of $1{ }^{\circ} \mathrm{C} / 5 \mathrm{~s}$ to $72{ }^{\circ} \mathrm{C}$, and 2 min at $72{ }^{\circ} \mathrm{C}$; and (3) a 10 -min incubation at $72{ }^{\circ} \mathrm{C}$, respectively. The PCR products were sequenced by Genfanavaran Biotech Corporation (O'Donnell et al. 2007). The DNA sequences determined for this study were submitted to GenBank, and the accession numbers for strain CBS $115.40=$ IBRCM 30232 are: KX503270 (RPB2), KX503269 (EF$1 \alpha)$, KX503267 (ITS). The accession numbers for strain CBS $139505=$ IBRC-M 30096 KX503268 (ITS).

Sequence analysis

Each of the DNA fragments was sequenced on both directions using the same primers which were used for PCR amplification. Sequences were assembled and edited with a trial version of Geneious software (www.geneious.com).
Using the MEGAv. 7.0.9 package, sequences were aligned with sequences obtained from the online databases ofCBS, NBRC, and GenBank (http://www.ncbi.nlm.nih.gov/). According to the results gained from the similarity assessments (CBS, Fusarium MLST, and NCBI), sequences were aligned with the multiple sequence alignment tool; Multiple sequence Alignment using Fast Fourier Transform (MAFFT), available at the European Bioinformatics Institute (EMBL-EBI) (Katoh et al. 2009, McWilliam et al. 2013). Alignments were manually improved in MEGA v. 7.0.9 and Bioedit v. 7.0.5.3 packages (default settings) (Tamura et al. 2011; Kumar et al. 2016). The flanking regions were excluded from the analysis. The alignments were checked visually and finally the resulting multiple sequence alignments were used for phylogenetic assessments. Concatenated multi-locus sequence alignments were prepared with the BioEdit 7.0.5.3 package. Phylogenetic trees were rooted with Fusarium staphyleae strain NRRL 22316. Phylogenetic analyses were performed for each dataset as well as with combined alignments consisting of ITS, EF- $1 \alpha$, and RPB 2 regions.

The online tool Findmodel (http://www.hiv.lanl. gov/content/sequence/findmodel/findmodel. html) was used to determine the best nucleotide substitution model. Maximum likelihood (ML) distance analysis was conducted with the MEGA v. 7.0.9 package (Tamura et al. 2011) with the GTR + GAMMA substitution models. The robustness of the trees was evaluated by 1000 bootstrap replications. Bayesian analyses were conducted with MrBayes v3.2.1 (Huelsenbeck and Ronqvist 2001) executed on XSEDE (Extreme Science and Engineering Discovery Environment) through the CIPRES Science Gateway v3.3 (Miller et al. 2010) in two parallel runs, using the default settings but with these adjustments: general time reversible (GTR) model of DNA substitution as the best fit and a gamma distribution rate variation across sites (Huelsenbeck and Ronqvist 2001). This model was chosen as the result from a pretest with MrModeltest 2.2 (Nylander 2004). After this was determined, the GTR + I + G model, as the best nucleotide substitution model, was used for the combined ITS, EF- $1 \alpha$, and RPB2 dataset, and a MCMC heated chain was set with a temperature value of 0.05 . The number of chains, number of generations, and sample frequencies were set respectively at 4, 50,000,000, and 1000. Chain convergence was determined using Tracer v1.5 (http://ree.bio.ed.ac.uk/software/tracer/) to confirm sufficiently large ESS values (>200). The sampled trees were subsequently summarized after omitting the first $25 \%$ of trees as burn-in using the "sump" and "sumt" 
commands implemented in MrBayes (Rambaut and Drummond 2009). Trees were visualized and edited using FigTree v1.4.2 (Rambaut 2008). The concatenated aligned dataset for ITS, EF-1 $\alpha$ and RPB2 used in the analysis has been submitted with the TreeBASE under the submission ID 21561 (Papizadeh et al. 2017b).

Phytopathogenicity tests

Strains of Fusarium ershadii (CBS 115.40 and CBS 139505) were grown on PDA for a week, the surface of the medium was removed using a sterile scalpel, and the mycelial material was added to $25 \mathrm{ml}$ of sterile $0.05 \%$ tween 80 solution. The suspension was vortexed for $20 \mathrm{~min}$ and then filtered through sterile cotton cloth to remove hyphae. Thereafter, conidia were counted using a haemocytometer and a suspension with an approximate density of $1.2 \times 10^{6} / \mathrm{ml}$ was prepared. Roots of 11 months old Asparagus officinalis (Accession IBRC P1006759 of the Iranian Biological Reference Centre) seedlings, grown in greenhouse $\left(25^{\circ} \mathrm{C}\right.$ and 12 -h photoperiod), were washed in sterile water. Then, the roots of different seedlings were inoculated by immersion for a minute in the suspension of the conidia of strains CBS 115.40 and IBRC-M 30096, respectively. All tests were done in triplicate. Triplicate uninoculated seedlings were used as control. Finally, all the seedlings were potted in sterilized-soil. Pots were incubated in a quarantined space in the greenhouse for three weeks and were examined and photographed in 3-day intervals. The same procedure was performed on two months old Musa acuminata (IBRC P1011416) seedlings produced by tissue culture.

\section{Results}

Phenotypic characterization

Strain CBS 115.40 was isolated by Bugnicourt in 1936 from Musa sapientum in Tonkin, Vietnam and was deposited in the CBS collection in 1940. The strain used to be the type strain of Cylindrocarpon tonkinense, a relative of Cylindrocarpon lichenicola. In 2002, Summerbell and Schroers showed that $C$. lichenicola falls within the FSSC, while it was noted that CBS 115.40 was a clearly distinct species also within the same Fusarium solani species complex (Summerbell and Schroers 2002). More recently CBS 139505 was isolated from diseased Aspagarus in Italy. Both strains proved to match in morphology and in multi-locus sequence analyses and we describe them here as Fusarium ershadii.

Morphological features of $F$. ershadii are shown in Table 1 and Fig. 2. Strains of F. ershadii, like other members of FSSC have septate, filiform conidiophores incorporating microconidia-bearing terminal monophialides. Although true macroconidia, characteristic of FSSC members, were not detected in dark nor under UV, some conidia, around $20 \mu \mathrm{m}$ in length and 3septate, were observed which may be assumed to be macroconidia (e.g. Fig $2 \mathrm{~g}$ and o). Chlamydospores were formed (e.g. Fig $2 \mathrm{~s}$ and $\mathrm{t}$ ), sometimes directly from the mostly 1-septate conidia.

Strains (CBS 115.40 and CBS 139505) showed the same macro- and micromorphology on agar plates with little to no pigmentation on the used media. Morphological characters are summarized in the species descriptions (Fig. 2). No growth was detected on MEA medium at $5{ }^{\circ} \mathrm{C}$. Hence, $10{ }^{\circ} \mathrm{C}$ was recorded as the lowest temperature that the strains could grow. The strains did not grow at $40{ }^{\circ} \mathrm{C}$ and after a period at temperatures of $42{ }^{\circ} \mathrm{C}$ or higher they lost viability and were unable to grow at growth-permitting temperatures. The optimum temperature for growth was between 28 and $30^{\circ} \mathrm{C}$. The optimum $\mathrm{pH}$ value for growth was 6 (Table 1).

Molecular Identification and phylogenetic analysis

Sequences of three loci, EF-1 $\alpha$, RPB2, and ITS fragments, of Fusarium ershadii were studied in combination with sequences of FSSC isolates already available in the Fusarium MLST and GenBank databases (Fig. 1). The sequence identity in EF- $1 \alpha$ fragments of 110 strains

Table 1 Growth profile of Fusarium ershadii

\begin{tabular}{llll}
\hline $\mathrm{pH}$ & Colony Diam. (mm) & Temp. & Colony Diam. (mm) \\
\hline 3 & No growth & 5 & No growth \\
3.5 & 12 & 10 & 7 \\
4 & 29 & 15 & 28 \\
5 & 46 & 20 & 39 \\
5.5 & 56 & 25 & 57 \\
6 & 58 & 28 & 59 \\
7 & 57 & 30 & 59 \\
7.2 & 56 & 34 & 22 \\
8 & 52 & 37 & 19 \\
8.5 & 43 & 40 & No growth \\
\hline
\end{tabular}


belonging to FSSC was about 56\% (pairwise identity $\sim 95 \%$ ). For the ITS fragment sequence identity was around $64-68 \%$ (pairwise identity $\sim 96.5 \%$ ) and for RPB2 fragment 70-71\% (pairwise identity $\sim 97.5 \%$ ).

Phylogenies performed on the combined set of ITS, EF- $1 \alpha$ and RPB2 fragments and individual fragments resulted similar tree topologies. The analyses placed F. ershadii into a distinct clade (MLST group FSSC 9c) (Fig. 1). As is shown in Fig. 1, the posterior probability support for the F. ershadii clade was $0.9992 / 97 \%$ ML bootstrap value. The remainder of the tree was similar to that described for the FSSC (O'Donnell et al. 1998) (Table 2).

\section{Phytopathogenicity}

We tested our two strains for pathogenicity on the two host plants they were isolated from. The same pathologic results were seen on the triplicate inoculated plants of Asparagus officinalis and Musa acuminata. Our phytopathogenicity tests showed that the inoculation of roots of Asparagus plants (Asparagus officinalis IBRC P1006759) with strains CBS 139505 and CBS 115.40 led to a severely reduced growth within 10 days (Figure. 3). Furthermore, the roots showed clearly 'hollow root'-like symptoms with strong pigmentation within root tissues and the formation of empty areas void of plant material. These symptoms are comparable to the symptoms of Fusarium crown and root rot in asparagus, normally attributed to Fusarium oxysporum f.sp. asparagi, $F$. proliferatum, unspecified $F$. solani, and $F$. redolens (Elmer 2015). In comparison, inoculation of roots of banana plants (Musa acuminata IBRC P1011416) with the same strains, resulted in a reduced growth but not to the level that was seen on Asparagus plants (Fig. 3d-e).

\section{Taxonomy}

Fusarium ershadii Papizadeh, van Diepeningen, \& Zamanizadeh, sp. nov. (Figs. 1 and 2).

Mycobank: MB 817602.

Type: Vietnam, Tonkin, isolated from Musa sapientum, 1936, collected by F. Bugnicourt. (holotype: IBRC-H 2025, a dried culture) [Ex-type: CBS $115.40^{\mathrm{T}}=$ IBRC-M $30232^{\mathrm{T}}$ preserved in a metabolically inactive state (cryopreserved)].

Additional strain: CBS 139505 = IBRC-M 30096.

Sequences from ex-type culture, CBS 115.40: ITS (KX503267), EF-1 $\alpha$ (KX503269), RPB2 (KX503270) and from strain CBS $139505=$ IBRC-M 30096: ITS (KX503268).
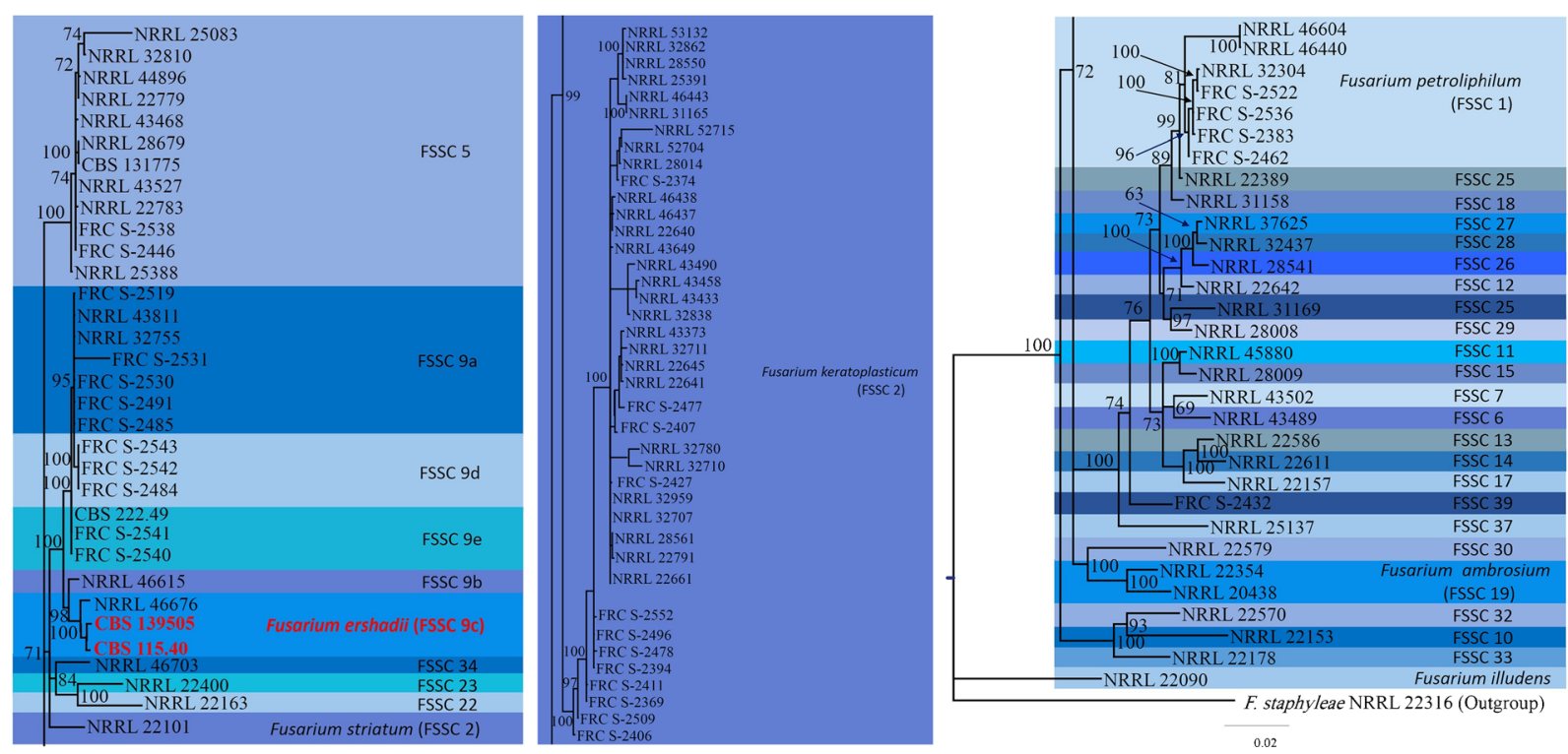

Fig. 1 Phylogenetic relationships with maximum likelihood and Bayesian inference methods under the GTR $+\mathrm{I}+\mathrm{G}$ model of evolution between Fusarium ershadii and the other members of FSSC based on the concatenated data of EF1- $\alpha$, ITS and RPB2 (ML tree shown). At the branch tips the strain identifiers are given.
In blocks species with latin binomials are indicated, but the majority of clades within FSSC do not have them yet. Clade 9c; Fusarium ershadii, Clade 5; Fusarium solani senso stricto, Clade $2 ;$ F. keratoplasticum, Clade 1; F. Petroliphylum. As outgroup Fusarium staphyleae NRRL 22316 was used 
Table 2 Strains and sequences used in this study

\begin{tabular}{|c|c|c|c|c|c|}
\hline \multirow[t]{2}{*}{ Species complexes of Fusarium } & \multirow[t]{2}{*}{ Strain number } & \multicolumn{3}{|c|}{ Sequence Accession Numbers } & \multirow[t]{2}{*}{ Reference } \\
\hline & & RPB2 & ITS & $\mathrm{EF} 1-\alpha$ & \\
\hline Fusarium ershadii $(9 \mathrm{c})$ & CBS 115.40 & KX503270.1 & KX503267.1 & KX503269.1 & This study \\
\hline Fusarium ershadii $(9 \mathrm{c})$ & CBS 139505 & & KX503268.1 & & This study \\
\hline Fusarium ershadii (9c) & NRRL 46676 & GU250731.1 & GU250669.1 & GU250546.1 & Balmas et al. (2010) \\
\hline FSSC 9b & NRRL 46615 & GU250728.1 & GU250666.1 & GU250543.1 & Balmas et al. (2010) \\
\hline FSSC 9d & FRC S-2484 & JN235906.1 & JN235291.1 & JN235721.1 & Short et al. (2011) \\
\hline FSSC 9d & FRC S-2542 & JN235907.1 & JN235292.1 & JN235722.1 & Short et al. (2011) \\
\hline FSSC 9d & FRC S-2543 & JN235908.1 & JN235293.1 & JN235723.1 & Short et al. (2011) \\
\hline FSSC 9a & FRC S-2519 & JN235911.1 & JN235296.1 & JN235726.1 & Short et al. (2011) \\
\hline FSSC 9a & FRC S-2485 & JN235909.1 & JN235294.1 & JN235724.1 & Short et al. (2011) \\
\hline FSSC 9a & FRC S-2491 & JN235912.1 & JN235297.1 & JN235727.1 & Short et al. (2011) \\
\hline FSSC 9a & FRC S-2530 & JN235910.1 & JN235295.1 & JN235725.1 & Short et al. (2011) \\
\hline FSSC 9a & FRC S-2531 & JN235913.1 & JN235298.1 & JN235728.1 & Short et al. (2011) \\
\hline FSSC 9a & NRRL 32755 & HM347159.1 & DQ094534.1 & DQ247073.1 & Zhang et al. (2006) \\
\hline FSSC 9a & NRRL 43811 & EF470092.1 & EF453204.1 & EF453053.1 & O'Donnell et al. (2007) \\
\hline FSSC 9e & CBS 222.49 & JX435259.1 & JX435209.1 & JX435159.1 & Debourgogne et al. (2012) \\
\hline FSSC 9e & FRC S-2540 & JN235914.1 & JN235299.1 & JN235729.1 & Short et al. (2011) \\
\hline FSSC 9e & FRC S-2541 & JN235915.1 & JN235300.1 & JN235730.1 & Short et al. (2011) \\
\hline FSSC 5 & FRC S-2446 & JN235917.1 & JN235302.1 & JN235732.1 & Short et al. (2011) \\
\hline FSSC 5 & FRC S-2538 & JN235942.1 & JN235327.1 & JN235757.1 & Short et al. (2011) \\
\hline FSSC 5 & CBS 131775 & JX237778.1 & JX162380.1 & JX118990.1 & Zhang et al. (2006) \\
\hline FSSC 5 & NRRL 28679 & EU329556.1 & DQ094385.1 & DQ246912.1 & Zhang et al. (2006) \\
\hline FSSC 5 & NRRL 43468 & EF469980.1 & EF453093.1 & EF452941.1 & O'Donnell et al. (2007) \\
\hline FSSC 5 & NRRL 22779 & EU329526.1 & DQ094333.1 & DQ246848.1 & Zhang et al. (2006) \\
\hline FSSC 5 & NRRL 32810 & EU329624.1 & DQ094577.1 & DQ247118.1 & Zhang et al. (2006) \\
\hline FSSC 5 & NRRL 25083 & JF740882.1 & JF741044.1 & JF740714.1 & O'Donnell et al. (2012) \\
\hline FSSC 5 & NRRL 44896 & GU170584.1 & GU170639.1 & GU170619.1 & Migheli et al. (2010) \\
\hline FSSC 5 & NRRL 22783 & EU329529.1 & DQ094335.1 & DQ246851.1 & O'Donnell et al. (2007) \\
\hline FSSC 5 & NRRL 43527 & EF470003.1 & EF453116.1 & EF452964.1 & O'Donnell et al. (2007) \\
\hline FSSC 5 & NRRL 25388 & EU329535.1 & DQ094341.1 & DQ246858.1 & Zhang et al. (2006) \\
\hline FSSC 22 & NRRL 22163 & EU329496.1 & AF178394.1 & AF178328.1 & O'Donnell et al. (2008) \\
\hline FSSC 23 & NRRL 22400 & EU329509.1 & DQ094303.1 & AF178343.1 & O'Donnell et al. (2008) \\
\hline FSSC 34 & NRRL 46703 & EU329661.1 & EU329712.1 & HМ347126.1 & O'Donnell et al. (2010) \\
\hline F. keratoplasticum (FSSC 2) & FRC S-2427 & JN235885.1 & JN235270.1 & JN235700.1 & Short et al. (2011) \\
\hline F. keratoplasticum (FSSC 2) & FRC S-2374 & JN235767.1 & JN235152.1 & JN235582.1 & Short et al. (2011) \\
\hline F. keratoplasticum (FSSC 2) & NRRL 28014 & EF470139.1 & DQ094354.1 & DQ246872.1 & Zhang et al. (2006) \\
\hline F. keratoplasticum (FSSC 2) & NRRL 43649 & EU329639.1 & EF453132.1 & EF452980.1 & O'Donnell et al. (2007) \\
\hline F. keratoplasticum (FSSC 2) & FRC S-2407 & JN235898.1 & JN235283.1 & JN235713.1 & Short et al. (2011) \\
\hline F. keratoplasticum (FSSC 2) & FRC S-2477 & JN235897.1 & JN235282.1 & JN235712.1 & Short et al. (2011) \\
\hline F. keratoplasticum (FSSC 2) & NRRL 22641 & EU329521.1 & DQ094328.1 & DQ246843.1 & Zhang et al. (2006) \\
\hline F. keratoplasticum (FSSC 2) & NRRL 22645 & EU329523.1 & DQ094330.1 & DQ246845.1 & Zhang et al. (2006) \\
\hline F. keratoplasticum (FSSC 2) & NRRL 52715 & JF741123.1 & JF740912.1 & JF740797.1 & O'Donnell et al. (2012) \\
\hline
\end{tabular}


Table 2 (continued)

\begin{tabular}{|c|c|c|c|c|c|}
\hline \multirow[t]{2}{*}{ Species complexes of Fusarium } & \multirow[t]{2}{*}{ Strain number } & \multicolumn{3}{|c|}{ Sequence Accession Numbers } & \multirow[t]{2}{*}{ Reference } \\
\hline & & RPB2 & ITS & $\mathrm{EF} 1-\alpha$ & \\
\hline F. keratoplasticum (FSSC 2) & NRRL 32711 & EU329597.1 & DQ094493.1 & DQ247031.1 & Zhang et al. (2006) \\
\hline F. keratoplasticum (FSSC 2) & NRRL 43373 & EF469959.1 & EF453072.1 & EF452920.1 & O'Donnell et al. (2007) \\
\hline F. keratoplasticum (FSSC 2) & NRRL 32780 & EU329617.1 & DQ094551.1 & DQ247090.1 & Zhang et al. (2006) \\
\hline F. keratoplasticum (FSSC 2) & NRRL 32959 & EU329634.1 & DQ094632.1 & DQ247178.1 & Zhang et al. (2006) \\
\hline F. keratoplasticum (FSSC 2) & NRRL 22640 & EU329520.1 & DQ094327.1 & DQ246842.1 & Zhang et al. (2006) \\
\hline F. keratoplasticum (FSSC 2) & NRRL 46437 & GU170588.1 & GU170643.1 & GU170623.1 & Migheli et al. (2010) \\
\hline F. keratoplasticum (FSSC 2) & NRRL 46438 & GU170589.1 & GU170644.1 & GU170624.1 & Migheli et al. (2010) \\
\hline F. keratoplasticum (FSSC 2) & NRRL 22661 & EU329524.1 & DQ094331.1 & DQ246846.1 & Zhang et al. (2006) \\
\hline F. keratoplasticum (FSSC 2) & NRRL 22791 & EU329530.1 & DQ094337.1 & DQ246853.1 & Zhang et al. (2006) \\
\hline F. keratoplasticum (FSSC 2) & NRRL 28561 & EU329552.1 & DQ094375.1 & DQ246902.1 & Zhang et al. (2006) \\
\hline F. keratoplasticum (FSSC 2) & NRRL 25391 & EU329536.1 & DQ094343.1 & DQ246860.1 & Zhang et al. (2006) \\
\hline F. keratoplasticum (FSSC 2) & NRRL 28550 & EU329547.1 & DQ094365.1 & DQ246891.1 & Zhang et al. (2006) \\
\hline F. keratoplasticum (FSSC 2) & NRRL 32862 & EU329631.1 & DQ094621.1 & DQ247167.1 & Zhang et al. (2006) \\
\hline F. keratoplasticum (FSSC 2) & NRRL 53132 & GU170598.1 & GU170654.1 & GU170634.1 & Migheli et al. (2010) \\
\hline F. keratoplasticum (FSSC 2) & NRRL 31165 & EU329562.1 & DQ094394.1 & DQ246921.1 & Zhang et al. (2006) \\
\hline F. keratoplasticum (FSSC 2) & NRRL 46443 & GU170591.1 & GU170646.1 & GU170626.1 & Migheli et al. (2010) \\
\hline F. keratoplasticum (FSSC 2) & NRRL 32707 & EU329595.1 & DQ094490.1 & DQ247027.1 & Zhang et al. (2006) \\
\hline F. keratoplasticum (FSSC 2) & NRRL 32710 & EU329596.1 & DQ094492.1 & DQ247030.1 & Zhang et al. (2006) \\
\hline F. keratoplasticum (FSSC 2) & NRRL 32838 & EU329627.1 & EU329681.1 & DQ247144.1 & Zhang et al. (2006) \\
\hline F. keratoplasticum (FSSC 2) & NRRL 43433 & DQ790561.1 & DQ790517.1 & DQ790473.1 & Chang et al. (2006) \\
\hline F. keratoplasticum (FSSC 2) & NRRL 43458 & EF470172.1 & EU329686.1 & DQ790511.1 & Chang et al. (2006) \\
\hline F. keratoplasticum (FSSC 2) & NRRL 43490 & DQ790573.1 & DQ790529.1 & DQ790485.1 & Chang et al. (2006) \\
\hline F. keratoplasticum (FSSC 2) & FRC S-2394 & JN235887.1 & JN235272.1 & JN235702.1 & Short et al. (2011) \\
\hline F. keratoplasticum (FSSC 2) & FRC S-2478 & JN235888.1 & JN235273.1 & JN235703.1 & Short et al. (2011) \\
\hline F. keratoplasticum (FSSC 2) & FRC S-2496 & JN235891.1 & JN235276.1 & JN235706.1 & Short et al. (2011) \\
\hline F. keratoplasticum (FSSC 2) & FRC S-2552 & JN235846.1 & JN235231.1 & JN235661.1 & Short et al. (2011) \\
\hline F. keratoplasticum (FSSC 2) & FRC S-2369 & JN235758.1 & JN235143.1 & JN235573.1 & Short et al. (2011) \\
\hline F. keratoplasticum (FSSC 2) & FRC S-2411 & JN235772.1 & JN235157.1 & JN235587.1 & Short et al. (2011) \\
\hline F. keratoplasticum (FSSC 2) & NRRL 52704 & JF741112.1 & JF740908.1 & JF740786.1 & O'Donnell et al. (2012) \\
\hline F. keratoplasticum (FSSC 2) & FRC S-2509 & JN235788.1 & JN235173.1 & $\mathrm{JN} 235603.1$ & Short et al. (2011) \\
\hline F. keratoplasticum (FSSC 2) & FRC S-2406 & JN235789.1 & JN235174.1 & $J \mathrm{~N} 235604.1$ & Short et al. (2011) \\
\hline F. striatum (FSSC 21) & NRRL 22101 & EU329490.1 & AF178398.1 & AF178333.1 & Chehri (2014) \\
\hline F. petroliphilum (FSSC 1) & FRC S-2383 & JN235858.1 & JN235243.1 & JN235673.1 & Short et al. (2011) \\
\hline F. petroliphilum (FSSC 1) & FRC S-2522 & JN235921.1 & JN235306.1 & JN235736.1 & Short et al. (2011) \\
\hline F. petroliphilum (FSSC 1) & NRRL 32304 & EU329568.1 & DQ094402.1 & DQ246932.1 & Zhang et al. (2006) \\
\hline F. petroliphilum (FSSC 1) & FRC S-2536 & JN235937.1 & JN235322.1 & JN235752.1 & Short et al. (2011) \\
\hline F. petroliphilum (FSSC 1) & FRC S-2462 & JN235938.1 & JN235323.1 & $\mathrm{JN} 235753.1$ & Short et al. (2011) \\
\hline F. petroliphilum (FSSC 1) & NRRL 46440 & GU170590.1 & GU170645.1 & GU170625.1 & Migheli et al. (2010) \\
\hline F. petroliphilum (FSSC 1) & NRRL 46604 & GU170594.1 & GU170649.1 & GU170629.1 & Migheli et al. (2010) \\
\hline FSSC 25 & NRRL 22389 & EU329506.1 & DQ094314.1 & AF178340.1 & Chehri (2017) \\
\hline FSSC 18 & NRRL 31158 & & DQ094389.1 & DQ246916.1 & Zhang et al. (2006) \\
\hline
\end{tabular}


Table 2 (continued)

\begin{tabular}{|c|c|c|c|c|c|}
\hline \multirow[t]{2}{*}{ Species complexes of Fusarium } & \multirow[t]{2}{*}{ Strain number } & \multicolumn{3}{|c|}{ Sequence Accession Numbers } & \multirow[t]{2}{*}{ Reference } \\
\hline & & RPB2 & ITS & $\mathrm{EF} 1-\alpha$ & \\
\hline FSSC 37 & NRRL 25137 & JF741084.1 & JF740899.1 & JF740757.1 & Sandoval-Denis et al. (2018) \\
\hline FSSC 29 & NRRL 28008 & EF470135.1 & DQ094350.1 & DQ246868.1 & Zhang et al. (2006) \\
\hline FSSC 25 & NRRL 31169 & KR673999.1 & DQ094396.1 & DQ246923.1 & Zhang et al. (2006) \\
\hline FSSC 26 & NRRL 28541 & EU329542.1 & EU329674.1 & DQ246882.1 & Zhang et al. (2006) \\
\hline FSSC 28 & NRRL 32437 & EU329581.1 & DQ094446.1 & DQ246979.1 & Zhang et al. (2006) \\
\hline FSSC 27 & NRRL 37625 & EU329637.1 & EU329684.1 & FJ240353.1 & O'Donnell et al. (2008) \\
\hline FSSC 12 & NRRL 22642 & EU329522.1 & DQ094329.1 & DQ246844.1 & Zhang et al. (2006) \\
\hline FSSC 39 & FRC S-2432 & JN235941.1 & JN235326.1 & JN235756.1 & Short et al. (2011) \\
\hline FSSC 7 & NRRL 43502 & DQ790576.1 & DQ790532.1 & DQ790488.1 & Chang et al. (2006) \\
\hline FSSC 15 & NRRL 28009 & EF470136.1 & DQ094351.1 & DQ246869.1 & Zhang et al. (2006) \\
\hline FSSC 11 & NRRL 45880 & EU329640.1 & EU329689.1 & FJ240352.1 & O'Donnell et al. (2008) \\
\hline FSSC 6 & NRRL 43489 & DQ790572.1 & DQ790528.1 & DQ790484.1 & Chang et al. (2006) \\
\hline FSSC 14 & NRRL 22611 & DQ094326.1 & EU329518.1 & DQ246841.1 & Zhang et al. (2006) \\
\hline FSSC 13 & NRRL 22586 & EU329516.1 & DQ094312.1 & AF178353.1 & O'Donnell et al. (2009) \\
\hline FSSC 17 & NRRL 22157 & EU329493.1 & DQ094306.1 & AF178359.1 & O'Donnell et al. (2009) \\
\hline FSSC 10 & NRRL 22153 & EU329492.1 & DQ094302.1 & AF178346.1 & O'Donnell et al. (2009) \\
\hline FSSC 32 & NRRL 22570 & EU329513.1 & AF178422.1 & AF178360.1 & O'Donnell et al. (2009) \\
\hline FSSC 33 & NRRL 22178 & EU329498.1 & DQ094313.1 & AF178334.1 & O'Donnell et al. (2009) \\
\hline F. ambrosium (FSSC 19) & NRRL 20438 & JX171584.1 & DQ094315.1 & AF178332.1 & O'Donnell et al. (2009) \\
\hline F. ambrosium (FSSC 19) & NRRL 22354 & EU329504.1 & DQ094316.1 & AF178338.1 & O'Donnell et al. (2009) \\
\hline FSSC 30 & NRRL 22579 & EU329515.1 & AF178415.1 & AF178352.1 & O'Donnell et al. (2009) \\
\hline F. illudens & NRRL 22090 & JX171601.1 & AF178393.1 & AF178326.1 & O'Donnell et al. (2009) \\
\hline F. staphyleae & NRRL 22316 & JX171609.1 & AF178423.1 & AF178361.1 & O'Donnell et al. (2009) \\
\hline
\end{tabular}

Etymology. Species epithet ershadii is selected in honor of Prof. Djafar Ershad for his contribution to mycology in Iran.

Conidia $12-20 \mu \mathrm{m}$ in length, thick-walled, $1-3$ septate oval, predominantly 1 -septate oval (k-m \& o), with little distinction in size between micro and macroconidia. Reniform microconidia rarely detected $(\mathrm{m})$. Conidiophores elongate $(50-130 \mu \mathrm{m})$, filiform, 1-3 septate, incorporating microconidia-bearing terminal monophialides (i \& j). Chlamydospores smooth-walled (p-s) or verrucoseverroculose (u), mostly intercalary, but also terminal. Singular intercalary chlamydospores globose to subglobose with or without supporting cells (4.5-7.5 $\mu \mathrm{m}$ in diam) (h, $\mathrm{q}$ $\&$ r). Pairs (n) and clusters of 2-4 celled globose to subglobose smooth-walled chlamydospores $(4.5-7.5 \mu \mathrm{m}$ in diam) with (p-w) or without supporting cells (s).

Culture characteristics- Colonies fast-growing, growth rate on oatmeal agar (OA), 0.45 cmday $^{-1}$; a-b.
Growth rate on potato dextrose agar (PDA), 0.43 cmday $^{-1}$; c-d. Growth rate on synthetic nutrient agar

Fig. 2 Morphological properties of Fusarium ershadii (strain CBS 115.40 and CBS 139505). a-b. Fast-growing 10-day old colony on oatmeal agar (OA) front and back side, growth rate approx. 0.45 cmday-1; c-d. Ten-day old colony on potato dextrose agar (PDA) front and back side, growth rate approx. 0.43 cmday-1; e-f. Tenday old colony on synthetic nutrient agar (SNA) front and back side, growth rate approx. 0.43 cmday-1: the strain has covered the whole plate area with thin mycelium; g. Germinating microconidium (PDA 1000×, cotton-blue stained); h. Single chlamydospore; i-j. Monophialidic conidiophores of aerial mycelium; K-1. 1-septate oval Microconidia, (PDA 1000×, cotton-blue stained); m. 1-septate reniform microconidium; n. pairs of chlamydospores; o. 1-3 septate oval microconidia; $\mathrm{p}-\mathrm{w}$ various forms of chlamydospores; $\mathrm{x}$. Chlamydospore formed on microconidium. All scale bars $10 \mu \mathrm{m}$ 

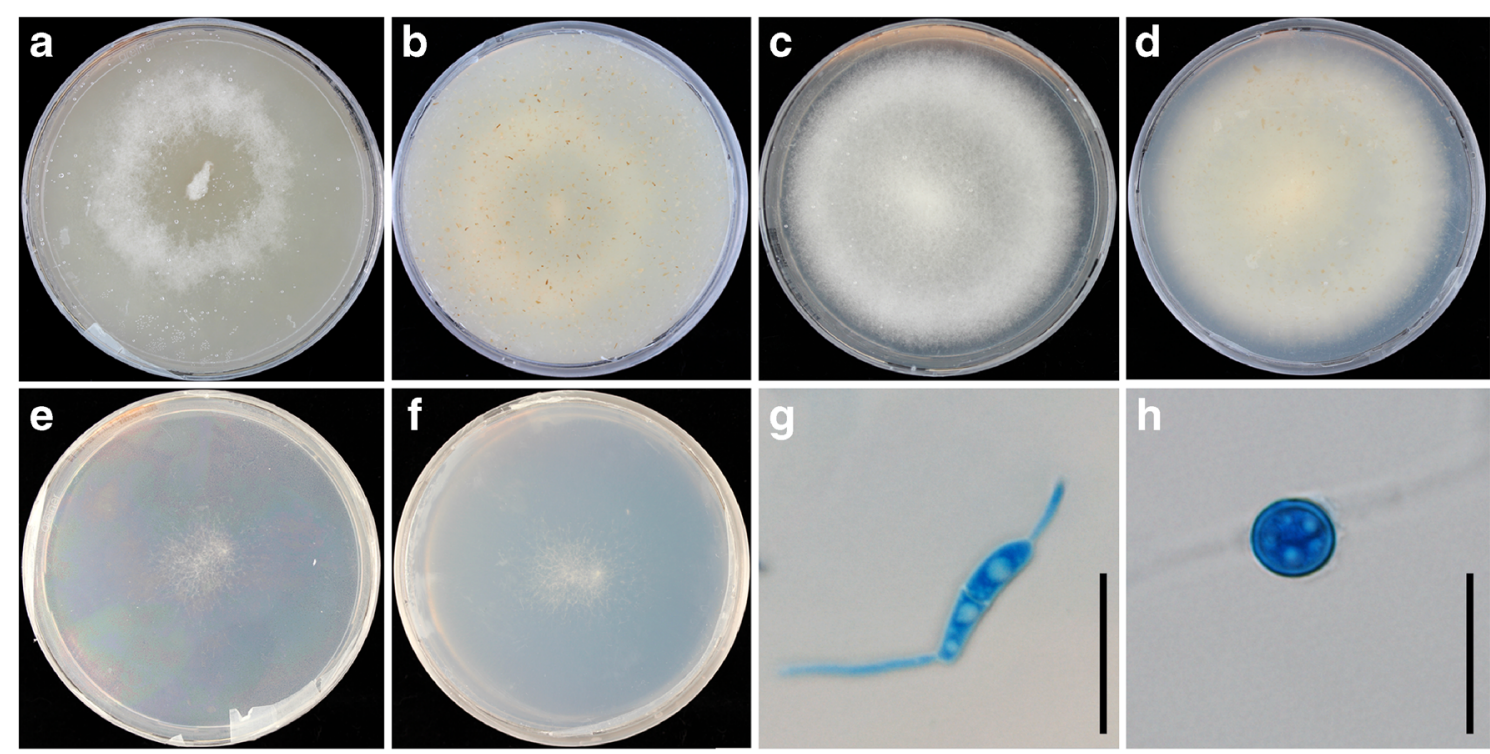

h
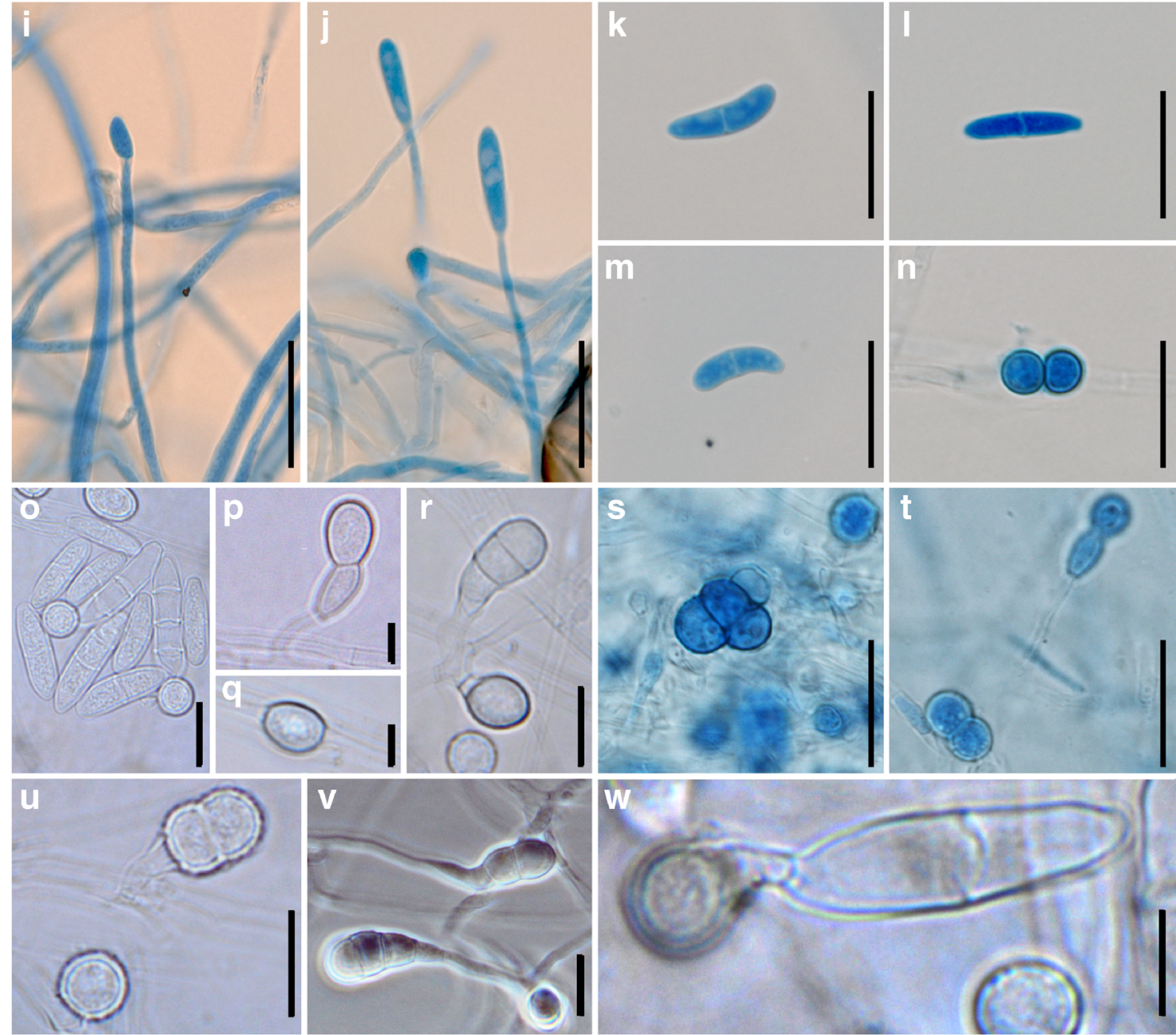

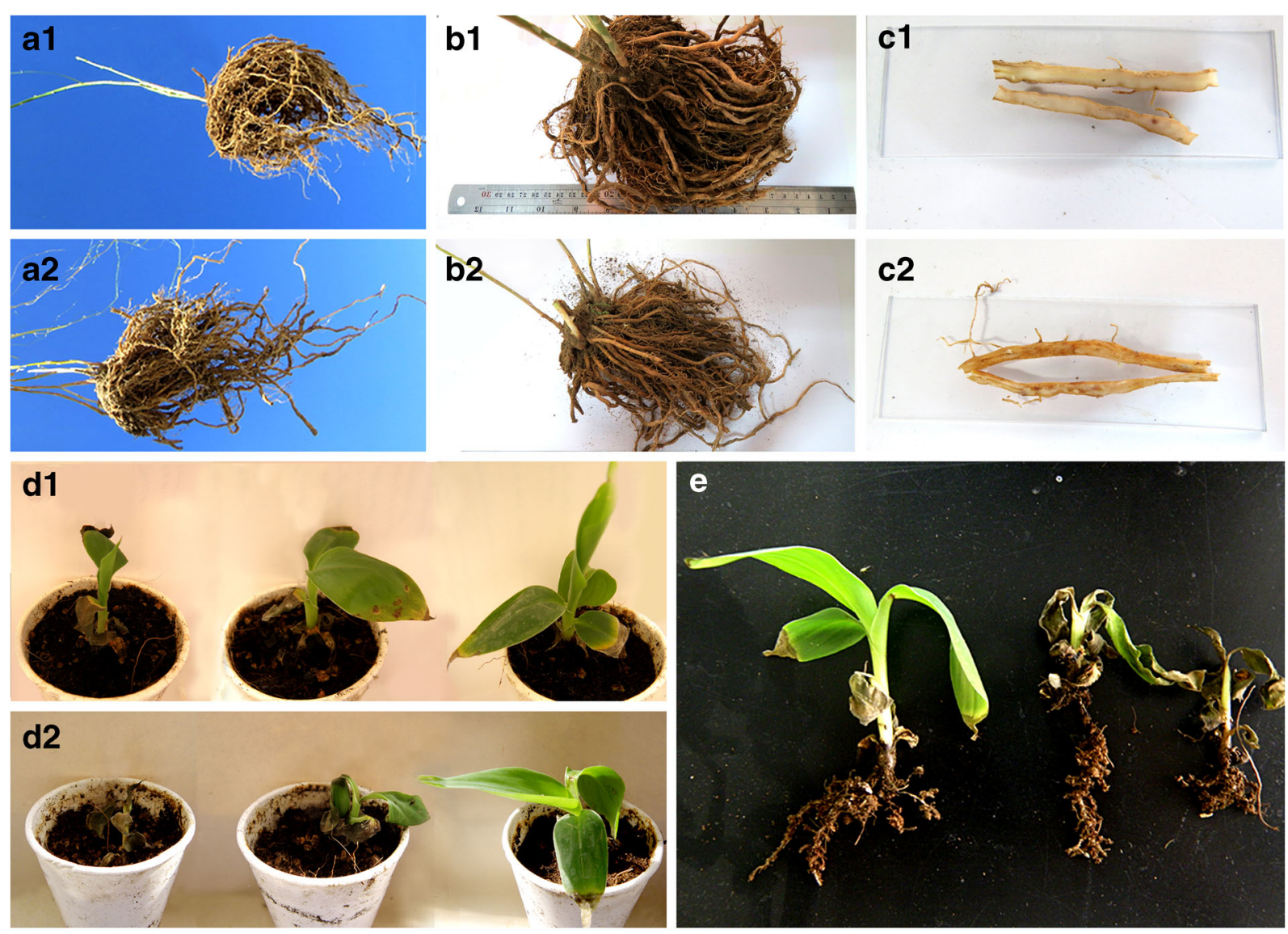

Fig. 3 Phytopathogenicity of Fusarium ershadii strains on Asparagus officinalis (A, B, and $\mathrm{C}$ ) and Musa acuminata (D and E). Hollow root disease symptoms in Asparagus officinalis induced by inoculation of Fusarium ershadii strains (un-inoculated; A1, $\mathrm{B} 1$, and $\mathrm{C} 1$, inoculated; A2, B2, and C2). Inoculation of the roots

(SNA) 0.43 cmday $^{-1}$; e-f. The strain has covered the Petri dish with thin mycelium. Colonies attaining $29 \mathrm{~mm}$ diam. in $7 \mathrm{~d}$ on PDA at $20^{\circ} \mathrm{C}, 57 \mathrm{~mm}$ diam. at $25^{\circ} \mathrm{C}, 59 \mathrm{~mm}$ diam. at $28-30{ }^{\circ} \mathrm{C}, 22 \mathrm{~mm}$ diam. at $34^{\circ} \mathrm{C}$, and $19 \mathrm{~mm}$ at $37^{\circ} \mathrm{C}$. Fungus did not grow at 5 and $40^{\circ} \mathrm{C}$ and it became unviable at $42{ }^{\circ} \mathrm{C}$. Incubating under an alternating day/night $12 \mathrm{~h}$ photoperiod. The fungus grew at a broad range of $\mathrm{pH}$ (3.5-8.5), while the optimum growth occurred at $\mathrm{pH}$ values between 5.5 and 6.5 .

\section{Discussion}

Fusarium ershadii forms a well-supported monophyletic lineage within clade 9 of FSSC and can be distinguished from all other species in this group based on DNA sequence comparisons and morphology. Fusarium ershadii of the Musa acuminata with strains of Fusarium ershadii. The pathogenicity test after one (D1) and two weeks (D2), from right; un-inoculated, inoculated with CBS 139505, and inoculated with CBS 115.40. Musa acuminata seedlings, from left; un-inoculated, inoculated with CBS 139505, and inoculated with CBS 115.40

seems to occur at least in North-America, Europe and Asia as a saprobe and as a pathogen. Here we have shown that the new species is a strong pathogen on Asparagus officinalis and a weak pathogen on Musa acuminata.

Genealogical concordance phylogenetic species recognition (GCPSR) (Taylor et al. 2000) is based on the concordance of multiple gene genealogies. In this study three loci; EF-1 $\alpha$, RPB2, and ITS, were used for GCPSR analyses, singly and concatenated. The EF- $1 \alpha$ and RPB2 fragments used have high levels of variation and are suitable barcodes for Fusarium (e.g. Al-Hatmi et al. 2016), while ITS is the general DNA barcode for fungi (Schoch et al. 2012), all three barcodes have the benefit that public repositories like Genbank contain large numbers of them for fungi of the genus Fusarium. Sequence analysis of the combined set of ITS, EF- $1 \alpha$ and RPB2 showed similar results gained from each 
fragment individually. Besides, according to the sequence identity and average pairwise identity values gained from the sequence analysis of EF- $1 \alpha$, RPB2, and ITS fragments, it can be inferred that the ITS fragment has a significant resolution in FSSC and it is quite different from what is seen in other species complexes of Fusarium (Papizadeh et al. 2015 and Papizadeh et al. 2016). Such a different resolution power of ITS fragment in FSSC conforms to the fact that FSSC forms a basal clade in the genus Fusarium with a significant phylogenic distance from the other clades of the genus. Additionally, although EF- $1 \alpha$ fragment, as the most variable fragment in FSSC, is recommended as the first choice for species delimitation in FSSC, the complementary effects of ITS and RPB-2 fragments should not be neglected, because they cause a higher robustness in phylogeny studies which can be inferred from the bootstrap and posterior probability values.

In general, the growth profile of $F$. ershadii seems to be similar to that of $F$. keratoplasticum (FSSC 2), F. petroliphilum (FSSC 1), and F. solani s.s. (FSSC 5) (Short et al. 2013; Schroers et al. 2016). The optimum temperature for growth of Fusarium ershadii is 28 $30{ }^{\circ} \mathrm{C}$, this temperature varies little in the described clades of FSSC (Short et al. 2013). Besides, growth rate studies on a $\mathrm{pH}$ gradient of showed that Fusarium ershadii can grow well in $\mathrm{pH}$ values between 3.5 and 8.5. However, the optimum $\mathrm{pH}$ was around 6 and a higher growth rate was recorded in mildly alkaline $\mathrm{pH}$ conditions (7.2-8.5) in comparison to mildly acidic $\mathrm{pH}$ values (4-5). While CBS 114.50 was first considered to be a close relative of $F$. lichenicola previously called Cylindrocarpon lichenicola (Summerbell and Schroers 2002), F. ershadii proves to be closer to the type species of $F$. solani than to $F$. lichenicola (lineage 16).

Morphologically, forming a diverse range of chlamydospores is a character which can be assumed as one of the main characteristics of FSSC. F. keratoplasticum and F. petroliphilum have also been described with such chlamydospores (Short et al. 2013). No sporodichia were detected to compare the morphology of sporodochial conidia of Fusarium ershadii to the other members of FSSC. Interestingly, aerial conidia of $F$. ershadii were 12-20 $\mu \mathrm{m}$ in length which is shorter than those of F. keratoplasticum, F. falciforme, F. petroliphilum and F. solani s.s. However, morphology and dimension of these conidia are highly similar to those of $F$. keratoplasticum.

Members of FSSC are cosmopolitan soil-borne hyphomycetes. These fusaria are also detected as pathogens not only of humans and other animals, but also a diverse range of plants. Based on sequence analyses, several additional strains were identified in Genbank as members of F. ershadii. The origins of these strains indicate a worldwide spread as saprobes and/or pathogens, as they include soil isolates from Sardinia, Italy (Balmas et al. 2010), a corn root isolate from Illionois, USA (Zhang et al. 2006), and a Chinese isolate from sugar beet (Cao and $\mathrm{Wu}$, unpublished results). Fusarium ershadii also has a special niche causing hollow root disease in Asparagus plants. It may have potential as a pathogen of other monocots such as banana and maize. Formae speciales, specialized on certain host crops in FSSC, are assumed to correspond to biologically and phylogenetically distinct species (Coleman 2015), whereas within the Fusarium oxysporum species complex host specificity encoded on supernumerary chromosomes were found to have been horizontally exchanged between different lineages and species (Baayen et al. 2000; Ma et al. 2013).

The Fusarium solani species complex is one of the more basal clades within the genus Fusarium according to the concept of Geiser et al. (2013); or, following Lombard et al. (2015) the clade would be called Neocosmospora (Lombard et al. 2015). The FSSC contain several members causing root rots and hollow roots and as Fusarium is the better known genus, we adhere to the first concept of a large monophyletic genus Fusarium and adhere to Fusarium ershadii for this new Asparagus pathogen.

Acknowledgements This work was performed at the Filamentous Fungi and Yeasts Collection, Microorganisms bank, IBRC and CBS-KNAW Fungal Biodiversity Centre, cooperatively. Great thanks for generous guides of Richard C. Summerbell, David M. Geiser and Kerry O'Donnell.

Open Access This article is distributed under the terms of the Creative Commons Attribution 4.0 International License (http:// creativecommons.org/licenses/by/4.0/), which permits unrestricted use, distribution, and reproduction in any medium, provided you give appropriate credit to the original author(s) and the source, provide a link to the Creative Commons license, and indicate if changes were made.

\section{References}

Al-Hatmi, A. M., van den Ende, A. H., Stielow, J. B., van Diepeningen, A. D., Seifert, K. A., McCormick, W., Assabgui, R., Gräfenhan, T., de Hoog, G. S., \& Levesque, 
C. A. (2016). Evaluation of two novel barcodes for species recognition of opportunistic pathogens in Fusarium. Fungal Biology, 120(2), 231-245.

Baayen, R. P., O'Donnell, K., Bonants, P. J., Cigelnik, E., Kroon, L. P., Roebroeck, E. J., \& Waalwijk, C. (2000). Gene Genealogies and AFLP Analyses in the Fusarium oxysporum Complex Identify Monophyletic and Nonmonophyletic Formae Speciales Causing Wilt and Rot Disease. Phytopathology, 90(8), 891-900.

Balmas, V., Migheli, Q., Scherm, B., Garau, P., O'Donnell, K., Ceccherelli, G., Kang, S., \& Geiser, D. M. (2010). Multilocus phylogenetics show high levels of endemic fusaria inhabiting Sardinian soils (Tyrrhenian Islands). Mycologia, 102(4), 803-812.

Chang, D. C., Grant, G. B., O'Donnell, K., Wannemuehler, K. A., Noble-Wang, J., Rao, C. Y., Jacobson, L. M., Crowell, C. S., Sneed, R. S., Lewis, F. M., Schaffzin, J. K., Kainer, M. A., Genese, C. A., Alfonso, E. C., Jones, D. B., Srinivasan, A., Fridkin, S. K., \& Park, B. J. (2006). Multistate outbreak of Fusarium keratitis associated with use of a contact lens solution. JAMA, 296(8), 953-963.

Chehri, K. (2014). Molecular phylogeny of the Fusarium solani species complex (FSSC) isolated from soils in Iran. Botany, 92(11), 815-820.

Chehri, K. (2017). Molecular identification of entomopathogenic Fusarium species associated with Tribolium species in stored grains. Journal of Invertebrate Pathology, 144, 1-6.

Coleman, J. J. (2015). The Fusarium solani species complex: ubiquitous pathogens of agricultural importance. Molecular Plant Pathology. https://doi.org/10.1111/mpp.12289.

Debourgogne, A., Gueidan, C., de Hoog, S., Lozniewski, A., \& Machouart, M. (2012). Comparison of two DNA sequencebased typing schemes for the Fusarium solani Species Complex and proposal of a new consensus method. Journal of Microbiological Methods, 91(1), 65-72.

Elmer, W. H. (2015). Management of Fusarium crown and root rot of Asparagus. Crop Protection, 73, 2-6.

Fisher, N. L., Burgess, L. W., Toussoun, T. A., \& Nelson, P. E. (1982). Carnation leaves as a substrate and for preserving cultures of Fusarium species. Phytopathology, 72, 151-153.

Geiser, D. M., Aoki, T., Bacon, C. W., et al. (2013). One Fungus, One Name: Defining the genus Fusarium in a scientifically robust way that preserves longstanding use. Phytopathology, 103, 400-408.

Huelsenbeck, J. P., \& Ronqvist, F. (2001). MrBayes: Bayesian inference of phylogeny. Bioinformatics, 17, 754-755. https://doi.org/10.1093/bioinformatics/17.8.754.

Hujslová, M., Kubátová, A., Kostovčík, M. \& Kolařík, M. (2013). Acidiella bohemica gen. et sp. nov. and Acidomyces spp. (Teratosphaeriaceae), the indigenous inhabitants of extremely acidic soils in Europe. Fungal Diversity, 58, 33-45.

Katoh, K., Asimenos, G. \& Toh, H. (2009). Bioinformatics for DNA sequence analysis. In Posada, D. (Ed.) Bioinformatics for DNA sequence analysis, methods in molecular biology, Methods in Molecular Biology (pp. 39-64). Totowa, NJ: Humana Press, Springer.

Kumar, S., Stecher, G. \& Tamura, K. (2016). MEGA7: Molecular Evolutionary Genetics Analysis version 7.0 for bigger datasets. Molecular Biology and Evolution, 33(7), 18701874 .
Lombard, L., van der Merwe, N. A., Groenewald, J. Z., \& Crous, P. W. (2015). Generic concepts in Nectriaceae. Studies in Mycology, 80, 189-245.

Ma, L. J., Geiser, D. M., Proctor, R. H., Rooney, A. P., O'Donnell, K., Trail, F., Gardiner, D. M., Manners, J. M., \& Kazan, K. (2013). Fusarium pathogenomics. Annual Review of Microbiology, 67, 399-416.

McWilliam, H., Li, W., Uludag, M., Squizzato, S., Park, Y.M., Buso, N., Cowley, A.P. \& Lopez, R. (2013). Analysis tool web services from the EMBL-EBI. Nucleic Acids Research, 41, 597-600.

Migheli, Q., Balmas, V., Harak, H., Sanna, S., Scherm, B., Aoki, T., \& O'Donnell, K. (2010). Molecular phylogenetic diversity of dermatologic and other human pathogenic fusarial isolates from hospitals in northern and central Italy. Journal of Clinical Microbiology, 48(4), 1076-1084.

Miller, M.A., Pfeiffer, W., Schwartz, T., (2010). Creating the CIPRES Science Gateway for inference of large phylogenetic trees in Proceedings of the Gateway Computing Environments Workshop (GCE), 14 Nov. 2010, New Orleans, LA. 1-8.

Nylander, J.A.A. (2004) MrModeltest 2. Program distributed by the author. Evolutionary Biology Centre. Uppsale: Uppsala Univ. AQ16

O’Donnell, K., Kistler, H. C., Cigelnik, E. \& Ploetz, R. C. (1998). Multiple evolutionary origins of the fungus causing Panama disease of banana: concordant evidence from nuclear and mitochondrial gene genealogies. Proceedings of the National Academy of Sciences of the United States of America, 95, 2044-2049.

O'Donnell, K., Sarver, B. A., Brandt, M., Chang, D. C., NobleWang, J., Park, B. J., Sutton, D. A., Benjamin, L., Lindsley, M., Padhye, A., Geiser, D. M., \& Ward, T. J. (2007). Phylogenetic diversity and microsphere array-based genotyping of human pathogenic Fusaria, including isolates from the multistate contact lens-associated U.S. keratitis outbreaks of 2005 and 2006. Journal of Clinical Microbiology, 45(7), 2235-2248.

O'Donnell, K., Sutton, D. A., Fothergill, A., McCarthy, D., Rinaldi, M. G., Brandt, M. E., Zhang, N., \& Geiser, D. M. (2008). Molecular phylogenetic diversity, multilocus haplotype nomenclature, and in vitro antifungal resistance within the Fusarium solani species complex. Journal of Clinical Microbiology, 46(8), 2477-2490.

O'Donnell, K., Sutton, D. A., Rinaldi, M. G., Gueidan, C., Crous, P. W., \& Geiser, D. M. (2009). Novel multilocus sequence typing scheme reveals high genetic diversity of human pathogenic members of the Fusarium incarnatum-F. equiseti and F. chlamydosporum species complexes within the United States. Journal of Clinical Microbiology, 47(12), 3851-3861.

O'Donnell, K., Sutton, D. A., Rinaldi, M. G., Sarver, B. A., Balajee, S. A., Schroers, H. J., Summerbell, R. C., Robert, V. A., Crous, P. W., Zhang, N., Aoki, T., Jung, K., Park, J., Lee, Y. H., Kang, S., Park, B., \& Geiser, D. M. (2010). Internet-accessible DNA sequence database for identifying fusaria from human and animal infections. Journal of Clinical Microbiology, 48(10), 3708-3718.

O'Donnell, K., Humber, R. A., Geiser, D. M., Kang, S., Park, B., Robert, V. A., Crous, P. W., Johnston, P. R., Aoki, T., Rooney, A. P., \& Rehner, S. A. (2012). Phylogenetic diversity of insecticolous fusaria inferred from multilocus DNA sequence 
data and their molecular identification via FUSARIUM-ID and Fusarium MLST. Mycologia, 104(2), 427-445.

Papizadeh, M., Soudi, M. R., Amoozegar, M. A., \& Shazadeh Fazeli, S. A. (2015). A Comparative Analysis on ITS, RPB-2 and EF $1 \alpha$ Fragments, Aiming Species Delimitation in Fusarium Solani Species Complex (FSSC). Current Medical Mycology., 1(S1), 33.

Papizadeh, M., Zamanizadeh, H. R., Zabihi, S. S., Soudi, M. R., Amoozegar, M. A., \& Shahzadeh Fazeli, S. A. (2016). Highlighted by GCPSR studies: is Fusarium solani a Fusarium yet? Iranian Plant Protection Congress., 22, 138.

Papizadeh, M., Roayaei Ardakani, M., \& Motamedi, H. (2017a). Growth-phase dependent biodesulfurization of dibenzothiophene by Enterobacter sp. strain NISOC-03. Pollution., 3, 101-111.

Papizadeh, M., Soudi, M. R., Amini, L., Wijayawardene, N. N., \& Hyde, K. D. (2017b). Pyrenochaetopsis tabarestanensis (Cucurbitariaceae, Pleosporales), a new species isolated from rice farms in north Iran. Phytotaxa., 297(1), 15-28.

Rambaut, A. (2008) FigTree v1.1.1: Tree figure drawing tool. Available: http://tree.bio.ed.ac.uk/software /figtree/. Accessed 20 June 2008.

Rambaut A, Drummond AJ. (2009). Tracer v1.5. Available from http://beast.bio.ed.ac.uk/Tracer.

Saba, F., Papizadeh, M., Khansha, J., Sedghi, M., Rasooli, M., Amoozegar, M. A., Soudi, M. R., \& Shahzadeh Fazeli, S. A. (2016). A rapid and reproducible genomic DNA extraction protocol for sequence-based identifications of archaea, bacteria, cyanobacteria, diatoms, fungi, and green algae. Journal of Medical Bacteriology., 5(3-4), 22-28.

Salah, H., Al-Hatmi, A. M., Theelen, B., Abukamar, M., Hashim, S., van Diepeningen, A. D., Lass-Florl, C., Boekhout, T., Almaslamani, M., \& Taj-Aldeen, S. J. (2015). Phylogenetic diversity of human pathogenic Fusarium and emergence of uncommon virulent species. Journal of Infection., 15(634453), 00264-00269. https://doi.org/10.1016/j. jinf.2015.08.011.

Sandoval-Denis, M., Guarnaccia, V., Polizzi, G., \& Crous, P. W. (2018). Symptomatic Citrus trees reveal a new pathogenic lineage in Fusarium and two new Neocosmospora species. Persoonia, 40, 1-25.

Schoch, C. L., Seifert, K. A., Huhndorf, S., Robert, V., Spouge, J. L., Levesque, C. A., Chen, W., \& Fungal Barcoding Consortium. (2012). Nuclear ribosomal internal transcribed spacer (ITS) region as a universal DNA barcode marker for Fungi. Proceedings of the National Academy of Sciences (PNAS), 109(16), 6241-6246.
Schroers, H. J., Samuels, G. J., Zhang, N., Short, D. P. G., Juba, J. H., \& Geiser, D. M. (2016). Epitypification of Fusisporium (Fusarium) solani and its assignment to a common phylogenetic species in the Fusarium solani species complex. Mycologia. https://doi.org/10.3852/15-255.

Selbmann, L., de Hoog, G.S., Zucconi, L., Isola, D., Ruisi, S., Gerrits, A.H.G., Ende, V.A., Ruibal, C., De Leo, F., Urzì, C. \& Onofri, S. (2008). Drought meets acid: three new genera in a dothidealean clade of extremotolerant fungi. Studies in Mycology, 61, 1-20.

Short, D. P., O'Donnell, K., Zhang, N., Juba, J. H., \& Geiser, D. M. (2011). Widespread occurrence of diverse human pathogenic types of the fungus Fusarium detected in plumbing drains. Journal of Clinical Microbiology, 49(12), 4264-4272.

Short, D. P., O'Donnell, K., Thrane, U., Nielsen, K. F., Zhang, N., Juba, J. H., \& Geiser, D. M. (2013). Phylogenetic relationships among members of the Fusarium solani species complex in human infections and the descriptions of $F$. keratoplasticum $s p$. nov. and F. petroliphilum stat. nov. Fungal Genetics and Biology, 53, 59-70.

Short, D. P., O'Donnell, K., \& Geiser, D. M. (2014). Clonality, recombination, and hybridization in the plumbing-inhabiting human pathogen Fusarium keratoplasticum inferred from multilocus sequence typing. BMC Evolutionary Biology, 14,91.

Summerbell, R. C., \& Schroers, H. J. (2002). Analysis of phylogenetic relationship of Cylindrocarpon lichenicola and Acremonium falciforme to the Fusarium solani species complex and a review of similarities in the spectrum of opportunistic infections caused by these fungi. Journal of Clinical Microbiology, 40(8), 2866-2875.

Tamura, K., Peterson, D., Peterson, N., Stecher, G., Nei, M. \& Kumar, S. (2011). MEGA5: Molecular evolutionary genetics analysis using maxi-mum likelihood, evolutionary distance, and maximum parsimony methods. Molecular Biology and Evolution, 28, 2731-2739.

Taylor, J. W., Jacobson, D. J., Kroken, S., Kasuga, T., Geiser, D. M., Hibbett, D. S., \& Fisher, M. C. (2000). Phylogenetic species recognition and species concepts in fungi. Fungal Genetics and Biology, 31(1), 21-32.

van Diepeningen, A. D., Al-Hatmi, A. M. S., Brankovics, B., \& de Hoog, G. S. (2014). Taxonomy and Clinical Spectra of Fusarium Species: Where Do We Stand in 2014? Current Clinical Microbiology Reports, 1, 10-18.

Zhang, N., O'Donnell, K., Sutton, D. A., Nalim, F. A., Summerbell, R. C., Padhye, A. A., \& Geiser, D. M. (2006). Members of the Fusarium solani species complex that cause infections in both humans and plants are common in the environment. Journal of Clinical Microbiology, 44(6), 2186-2190. 